\title{
Highlights of the scientific meeting of the 18th Annual Congress of the European Society of Skeletal Radiology (ESSR) 2011
}

\author{
Rob Campbell
}

Published online: 12 November 2011

(C) ISS 2011

\section{Introduction}

The 18th Annual Congress of the ESSR was hosted in Greece, on the beautiful island of Crete, 9-11 June 2011, with nearly 500 registrants present. The congress president was Prof Apostolos Karantanas. The meeting followed the same format as previous years with a musculoskeletal ultrasound course on the first day. The remaining 2 days of the meeting comprised the refresher course which concentrated on the topic of bone marrow disorders, with further focus sessions including arthritis, intervention, sports imaging, tumours and advances in MSK imaging. Fifty papers were accepted for presentation in the scientific sessions, with a further 77 papers accepted for the electronic poster exhibition. The abstracts for the scientific papers were published in the June 2011 edition of Skeletal Radiology [1]. This article summarises the highlights of the scientific program.

\section{Joint imaging}

This year's ESSR/ISS prize-winning abstract by Dietrich et al. described the normal variant of the supra-acetabular fossae (SAF) in the hip, which was found in $10 \%$ of MR studies and subsequently confirmed at arthroscopy. Two types are described: SAF-1, which fills with contrast on arthrography, and SAF-2, which is filled with cartilage. Knowledge of these lesions can prevent misdiagnosis of an osteochondral defect [2]. On a similar theme of normal

R. Campbell $(\bowtie)$

Department of Radiology, Royal Liverpool University Hospital, Liverpool L7 8XP, UK

e-mail: rob.campbell@rlbuht.nhs.uk variants, the different ossification patterns encountered in the femoral condyles of children and adolescents were described [3]. These lesions should not be confused with pathological processes such osteo-chondritis dissecans, a topic that was also discussed by Professor Klaus Bohndorf in the Founders lecture at the ISS meeting in San Diego, 2011.

A variety of other papers were presented focusing on imaging technique and applications. A small pilot study of T2* relaxation times of the menisci of the knee showed correlation with subjective grading scores for meniscal degeneration. However, further studies are required to determine if this finding is predictive of future meniscal tears [4]. Other technique-based abstracts assessed the reliability of 3D imaging for assessment of joints. Fast 3D MRI of the knee with PD SPAIR sequence was shown to be sensitive and specific for meniscal and ligament injuries compared with arthroscopy as a gold standard [5]. A normal volunteer study of foot MRI demonstrated that 3D SPACE imaging with multi-planar reformats is superior to three-plane orthogonal proton density MR imaging in demonstration of the Lisfranc ligaments [6]. The use of such 3D sequences with multi-planar reformation is becoming part of routine practice and, when used to replace standard sequences, allows for significant time savings in MR examinations.

\section{Tumours}

A retrospective review of 195 patients with chondrosarcoma (CS) assessed the impact of whole-body bone scintigraphy on initial surgical staging [7]. No cases of skeletal metastases were recorded in the study group. Multifocal CS was diagnosed in three patients. However, in two cases this was diagnosed on the initial MR staging exam, and in the third 
case this had a limited impact on the surgical staging. The authors concluded that routine whole-body isotope bone scanning has no role in initial tumour staging for CS.

Two studies addressed the perennial difficulties of distinguishing benign from malignant soft tissue tumours. One paper assessed the influence of myxoid matrix on the ADC values of tumours on diffusion-weighted MR imaging at $3 \mathrm{~T}$. The results suggest that although ADC values alone do not distinguish benign from malignant lesions, there are statistically significant differences between the ADC values of benign and malignant tumours when the myxoid and non-myxoid sub-groups are analysed independently [8]. However, conventional radiological interpretation remains the most useful for evaluating soft tissue masses. In a retrospective review of MR imaging of 138 benign and malignant superficial soft tissue masses, the authors demonstrated a significant relationship between malignancy and tumour lobulation, hemorrhage, fascial edema and necrosis. However, tumour size alone was not a predictive factor [9].

The winner of the ESSR Tumor Prize this year was awarded to Tsifountoudis et al. for their pictorial review of tumour and tumour-like lesions of Hoffa's fat pad, which was presented in the electronic poster exhibition. The article combined excellent MR images with arthroscopic and histological illustrations [10].

\section{Rheumatology}

Arthritis was a popular theme with several papers presented on the subject of contrast-enhanced US of synovitis (CEUS). Wick et al. demonstrated good to excellent interobserver agreement for a subjective four-point scoring system of synovial thickness and intensity of vascularisation. There was also excellent correlation with an objective quantification system calculating signal intensity on CEUS [11]. Another study showed that CEUS time intensity curves can reliably quantify synovitis and that "time to peak" and "area under the curve" calculations correlate with clinical and biological signs of inflammation [12]. CEUS was also shown to be significantly more sensitive than power Doppler US (PDUS) in demonstrating peri- and intra-tendinous vascularity in wrist tenosynovitis, with excellent interobserver variation utilising a three-point grading system [13]. Such results are encouraging and give credence to the use of CEUS as an alternative image modality to MRI for longitudinal studies in arthritis.

Two studies evaluated the use of contrast-enhanced MRI (CEMRI). Platzgummer et al. showed that half dose Gd (Multihance) correlates well with full dose CEMRI and can sufficiently depict synovitis in early rheumatoid arthritis [14]. In the second paper, diffusion-weighted MRI correlated well with CEMRI for depiction of acute entheseal lesions in ankylosing spondylitis. However, it is noteworthy that all lesions were also depicted as high SI on STIR imaging without contrast [15].

Cartilage imaging remains a popular avenue of research in arthritis. 3D deformation maps of articular cartilage in MRI of the knee following standardised loading regimes can show predictable deformation patterns [16]. Such techniques may contribute to the development of biomechanical models of the knee and help to predict stress-induced degeneration and osteoarthritis.

\section{Osteoporosis and bone marrow imaging}

Several papers were presented that reflected the main subject matter of the refresher course. Two studies described the potential use of MR spectroscopy in quantifying bone marrow fat content. A study of 80 volunteers evaluating $1 \mathrm{H}$ spectroscopy of hips in male and female subjects showed that the fat content differed predictably across various sites around the hip [17]. The spectroscopic conversion index showed a statistically significant difference between men and women in most age groups, but further studies are required to assess the value of this technique for pathological conditions around the hip. In another paper, the association between marrow fat content, abdominal adipose tissue, lumbar spine bone mineral density (BMD) and blood biomarkers in females with type II diabetes mellitus was assessed using proton spectroscopy (1H-MRS). A high correlation was noted between vertebral bone marrow fat and $\mathrm{HbAlc}$, suggesting that bone marrow fat may be a biomarker for the extent of glycemic control in diabetics [18].

Fracture prediction in osteoporosis was addressed in two papers using quantitative CT. A longitudinal study showed that BMD derived from sagittal reformations of the spine from routine contrast-enhanced CT of the abdomen could predict incidental osteoporotic fractures of the spine [19]. A smaller study of volumetric BMD using high resolution quantitative $\mathrm{CT}$ suggested that a different pattern of bone loss occurs in lung transplant patients than that found in patients with typical post-menopausal or age-related osteoporosis [20]. Optical flow deformation models allow recognition of morphological features such as trabecular thickness and spatial re-organisation. In the future computer-based analysis may help predict osteoporotic fracture in high-risk populations.

\section{Tendon and soft tissue disease}

A work in progress of sonoelastography in patients with Charcot arthropathy showed progressive loss of elasticity 
over time in the plantar fascia of patients affected by diabetes mellitus [21]. However, it has yet to be shown whether these changes pre-date the radiological manifestations of arthropathy. Another study suggested that sonoelastography of the plantar fascia correlates favourably with conventional US in diagnosis of plantar fasciitis [22]. However, the clinical impact of sonoelastography remains controversial.

A 5-year longitudinal study of patellar and Achilles tendon in asymptomatic elite rugby players demonstrated that focal hypoechoic lesions and areas of microcalcification present on initial imaging all progressed in size and number at 5 year follow-up [23]. These findings emphasise the need to carefully correlate radiological and clinical findings.

A wide spectrum of other abstracts were also presented during the scientific sessions over the course of the ESSR meeting, including several papers on interventional techniques, and MRI and US imaging of other joint and soft tissue pathology. Further details are available for review in the published abstracts [1].

\section{Prize-winning presentations and E posters}

\section{ESSR/ISS Prize for Best Scientific Paper}

- TJ Dietrich et al. Switzerland. Supra-acetabular fossa (pseudo-defect of acetabular cartilage): morphology at MR arthrography

\section{ESSR Tumor Prize}

- I Tsifountoudis et al. Thessaloniki, Greece. MR imaging of tumors and tumor-like lesions of Hoffa's fat pad

\section{Poster Prizes}

- P. Mercouris. Durban, South Africa. MRI of SLAP lesions: a pictorial review of imaging and anatomy with arthroscopic correlation

- E. Fabbro et al. Genoa, Italy. Dynamic ultrasound evaluation (HRUS) of the hip: a detailed didactic approach

- V. Skiadis et al. Athens, Greece. Anterior knee pain. A pictorial essay

\section{References}

1. 18th Annual Congress of the European Society of Skeletal Radiology (ESSR), Crete, Greece, June 9-11, 2011. Skeletal Radiol. 2011;40:793-814

2. Dietrich TJ, Suter A, Pfirrmann C, Dora C, Fucentese SF, Zanetti M. Supra-acetabular fossa (pseudo-defect of acetabular cartilage): morphology at MR-arthrography. Skeletal Radiol. 2011;40:806-7.
3. Jans LBO, Jaremko JL, Ditchfield M, Verstraete KL. Femoral condylar ossification variants at MR imaging: types, frequency and age distribution. Skeletal Radiol. 2011;40:795.

4. Noebauer-Huhmann I, Welsch GH, Juras V, Szomolanyi P, Trattnig S, Marlovits S, et al. Quantitative biochemical T2* MRI of menisci at $3 \mathrm{~T}$ and correlation with morphological grading. Preliminary results. Skeletal Radiol. 2011;40:809.

5. McDonald S, Mckean D, Teoh E, McNally E. Fast 3D MRI of the knee: experience in clinical practice. Skeletal Radiol. 2011;40:812.

6. Ulbrich EJ, Zubler V, Sutter R, Espinosa N, Pfirrmann C, Zanetti M. Ligaments of the Lisfranc joint: comparison 3D (SPACE=sampling perfection with application optimized contrasts using different flip angle evolution) MR sequence and three orthogonal proton-density fat saturated (PD fs) MR sequences. Skeletal Radiol. 2011;40:807.

7. Douis H, James SL, Grimer RJ, Davies MA. Is bone scintigraphy necessary in the initial surgical staging of chondrosarcoma of bone? Skeletal Radiol. 2011;40:801.

8. Noebauer-Huhmann I, Krssak M, Amann G, Panotopoulis J, Funovics P, Fruehwald-Pallamar J, et al. Soft tissue tumors at 3 Tesla MRI: influence of a myxoid matrix on diffusion weighted imaging. Skeletal Radiol. 2011;40:811.

9. Calleja M, Dimegan M, Saifuddin A. MRI of soft tissue masses: analysis of features useful in distinguishing between benign and malignant lesions. Skeletal Radiol. 2011;40:807-8.

10. Tsifountoudis I, Kalaitzoglou I, Patsinakidis N, Haritandi A, Kosmatopoulos F, Metaxa L, Dimitriadis AS. MR imaging of tumors and tumor-like lesions of Hoffa's fat pad. EPOS 18th Annual Congress of the European Society of Skeletal Radiology (ESSR), Crete, Greece, June 9-11, 2011. http:// posters.webpages.com/essr/e-poster/

11. Wick MC, Franz M, Jaschke W, Mur E, Gruber J, Klauser AS. Contrast-enhanced ultrasonography in the detection of joint vascularity-subjective grading versus objective quantification. Skeletal Radiol. 2011;40:793-4.

12. Cyteval C, Mouterde G, Hoa D, Morel J, Combe B. Factors influencing quantitative values of contrast-enhanced ultrasound and correlations with clinical values in rheumatoid arthritis. Skeletal Radiol. 2011;40:797.

13. Faschningbauer R, Guerra F, Gruber J, Feuchtner GM, Jaschke W, Klauser AS. Detection of vascularity in wrist tenosynovitis: power Doppler ultrasound compared to contrast-enhanced grey-scale ultrasound. Skeletal Radiol. 2011;40:798-9.

14. Platzgummer H, Lodemann K, Schueller G, Johannes G, Kainberger F, Weber M, et al. Contrast-enhanced MR imaging of hand and finger joints in patients with early rheumatoid arthritis: do we really need the full dose of Multihance for the assessment of synovial enhancement? Skeletal Radiol. 2011;40:804-5.

15. Sanal HT, Yilmaz S, Kalyoncu U, Cinar M, Simsek I, Erdem $\mathrm{H}$, et al. Diffusion-weighted MRI in assessing active sacroiliitis: can it replace contrast-enhanced sequences? Skeletal Radiol. 2011;40:799.

16. Horng A, Raya J, Zscharn M, Hoehne-Hueckstaedt U, Hermanns I, Glitsch U, et al. In-vivo cartilage deformation pattern of human knees after loading - a 3D-volumetric approach to identify potential contact areas. Skeletal Radiol. 2011;40:814-5.

17. Pansini VM, Monnet A, Penel G, Salleron J, Migaud H, Cotton A. Hip bone marrow $1 \mathrm{H}$-spectroscopy in a healthy population: assessment of normal characteristics and influence of age and sex with 3 Tesla MRI. Skeletal Radiol. 2011;40:795-6.

18. Baum T, Yap SP, Karampinos DC, Nardo L, Burghardt AJ, Li X, et al. Association of vertebral bone marrow fat content with abdominal adipose tissue, lumbar spine BMD and blood markers in women with type 2 diabetes mellitus. Skeletal Radiol. 2011;40:811. 
19. Baum T, Muller D, Dobritz M, Rummeny EJ, Link TM, Bauer J. Converted BMD values derived from sagittal reformations of contrast-enhanced MDCT predict incidental osteoporotic vertebral fractures. Skeletal Radiol. 2011;40:810.

20. Fischer L, Valentinitsch A, Deutschmann J, Schueller-Weidekamm C, Zweytick B, Aigner C, et al. Quantification of trabecular dynamics in HRpQCT images of lung transplant patients using optical flow techniques. Skeletal Radiol. 2011;40:800-1.

21. Samuelsson E, Aurell Y. Work in progress: changes in the connective tissue of the foot in patients with diabetes and
Charcot's disease. A prospective ultrasound study including sonoelastography and Doppler. Skeletal Radiol. 2011;40:793.

22. Orlandi D, Sconfienza LM, Fabbro E, Ferrero G, Martini C, Silvestri E. Sonoelastography (RT-E) of plantar fascia: comparison among healthy subjects and patients affected by plantar fasciitis. Skeletal Radiol. 2011;40:808.

23. Ferrero G, Sconfienza LM, Fabbro E, Orlandi D, Martini C, Silvestri E. Five-year ultrasound survey of functional overload injuries of patellar and Achilles tendons in elite rugby players. Skeletal Radiol. 2011;40:809. 